\title{
ON SYSTEMS OF STRICT IMPLICATION
}

\author{
NAOTO YONEMITSU
}

(Received May 1,1950)

Introduction. Lewis attempted to construct a logistic system ${ }^{1)}$ containing an implication-relation $\prec$ such that $p \prec q$ ( $p$ strictly implies $q$ ) is synonymous with " $q$ is deducible from $p$ ". In his calculus, the propositions,

19.74

$\sim \diamond p \cdot \prec \cdot p \prec q$,

that is, if $p$ is impossilbe then $p$ strictly implies any proposition, and

19.75

$\sim \diamond \sim p \cdot \prec \cdot q \prec p$,

that is, if $p$ is necessary then any proposition $q$ strictly implies $p$, and further some paradoxical propositions have been proved ${ }^{2}$. $\mathrm{Emch}^{3}$ and Vredenduin ${ }^{4}$ showed that such paradoxical propositions should not always hold in usual logic, and they attempted in two different points of view, to construct new systems, the implication-relations of which seem to accord with the usual deducibility. Vredenduin accepts only $p \prec q \cdot \prec \cdot \diamond(p \sim q)$ as a postulate, but not $\sim \diamond(p \sim q) \cdot \prec \cdot p \prec q$, then he assumes $\prec$ as an undefined term. On the other hand, Emch assumes a unary operation $\bigcirc$ as an undefined term, by which he defines his implications $\sim n$, and develops his system in the analogous way to Lewis.

It is the purpose of this paper to present, in I some investigation of Vredenduin's suggestions, and certain properties in his system according to Mckinsey's results ${ }^{5}$, in II the equivalence between Vredenduin's system and Emch's one, and in III certin extensions of their systems from a viewpoint of modality.

I. Vredenduin's calculus of propositions is as follows: Undefined ideas; elementary propositions $p, q, r$, etc., negation $\sim p$, possibility $\diamond p$, product $p q$ or $p \cdot q$, implication $p \prec q$, and equivalence $p=q$.

1) Lewis AND LangFond, Symbolic Logic.

2) Op. cit., p. 248.

3) A. F. Fмсн, Implication and deducibility, Journ. of Symbolic Logic, vol. 1 (1936), pp. 26-35; Addendum to this paper, op. cit. p. 58.

C. I. Lewis, Emch's calculus and strict implication, op. cit., pp. 77-86.

A. F. EMcH, Deducibility with respect to necessary and impossible propositions, op. cit., vol. 2 (1937), pp. 78-81.

4) P. G. I. VRedendun, A system of strict implication, op cit, vol.14 (1939), pp. $73-76$.

5) J.C.C.Mckinsey, On the number of complete extensions of the Lewis's system, op. cit., vol. 9 (1944), pp. $42-45$.

J. C. C. Mckinsey, proof that there are infinitely many modalities in S2, op. cit., vol. 5 (1940), pp. $110-112$. 
Postulates ;

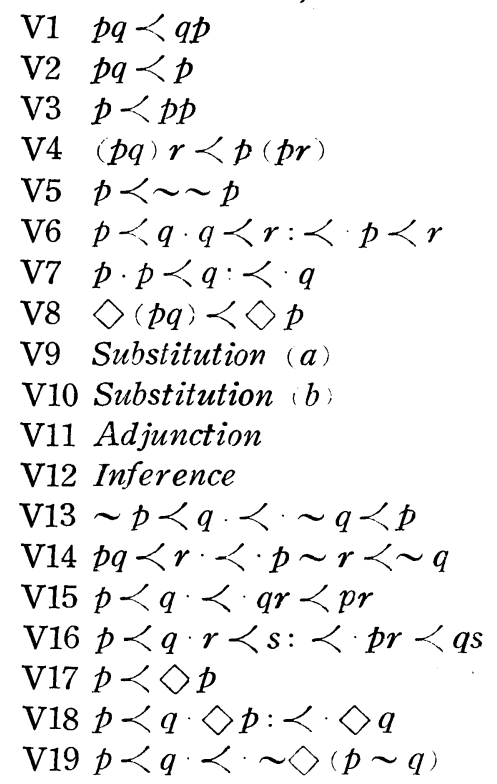

Definitions ;

V01 $p \vee q=\cdot \sim(\sim p \sim q)$

$\mathrm{V} 02 \quad p=q \cdot=\cdot p \prec q \cdot q<p$

V03 $p \supset q=\sim(p \sim q)$

$\mathrm{V} 04 \quad p \equiv q=p \supset q \cdot q \supset p$

$\mathrm{V} 05 p \circ a=\diamond(p q)$

1. It is obvious that this system is included in Lewis's system S2. Vredenduin states in his paper that the asserted propositions $17.51,17.52$ and 19. 47 of S2 can not be deduced from his assumptions, but if it were, 16.33, 16. $34,17.5,19.46,19.48,19.49,19.5,19.51$ and 19.52 could not be deduced because any of them can deduce some of 17.51, 17.52 and 19.47 in his calculus. (See the later proofs of 17.51 etc.) We are sure that these propositions have no paradoxical structure. In the following, we will show that they are all deducible in his calculus. The head numbers of propositions shall be identical with those in Symbolic Logic.

$12.1-16.32$ and 16.4-16.86 are proved in similar way in Symbolic Logic.

LEMMA 1. $p \prec q \cdot \prec: p \vee r \cdot \prec q \vee r[\mathrm{~V} 15,12.44, \mathrm{~V} 01,12.3]$

LEMMA 2. $p \prec q \cdot r \prec s: \prec: p \vee r \cdot \prec \cdot q \vee s$ [V16, 12.44, V01, 12.3]

LEMMA 3. $p \sim p \cdot \prec \cdot q, \quad p \cdot \prec \cdot q \vee \sim q$

[V14] $p q \prec p: \prec: p \sim p \cdot \prec \cdot \sim q$

[V 2] QED.

LEMMA 4. $p \cdot=\cdot p(q \vee \sim q), \quad p \cdot=\cdot p \vee(q \sim q)$

[V15] $p \cdot \prec \cdot q \vee \sim q: \prec: p p \cdot \prec \cdot(q \vee \sim q) p$

[LEM. 3, 12.7, 12.15] $p \cdot \prec \cdot p(q \vee \sim q)$

[V 2$]$

$p(q \vee \sim q) \cdot \prec \cdot p$

$[(1),(2), \mathrm{V} 02]$

LEMMA 5. $p \cdot \prec \cdot q \vee r:=: \sim r \cdot \prec \cdot q \vee \sim p[12.6,12.44,12.3, \mathrm{~V} 01, \mathrm{~V} 2]$

16.33

$p \prec q=\cdot p \prec p q$

[V15] $\quad p \prec q \cdot \prec p \prec q p$ 
$[12.7,12.15] \quad p \prec q \cdot \prec \cdot p \prec p q$

[LEM. 1] $\quad p \prec p q \cdot \prec: p \vee \sim p \cdot \prec \cdot p q \vee \sim p$

[16.73] $\quad p \prec p q \cdot \prec: p \vee \sim p \cdot \prec \cdot(p \vee \sim p)(q \vee \sim p)$

[LEM. 4] $\quad p \prec p q \cdot \prec: p \vee \sim p \cdot \prec \cdot q \vee \sim p$

[LEM. 5] $\quad p \prec p q \cdot \prec: p \cdot \prec \cdot q \vee(p \sim p)$

[LEM. 4] $\quad p<p q \cdot<: p \prec q$

$[(1),(2)]$

16. 34

16. 35 QED.

[13.2] $p \prec q:=: p \vee q \cdot \prec \cdot q \quad[16.33,12.44]$

[16.33] $p \cdot=: p \vee q \cdot p$

[V 2$]$

$$
\begin{aligned}
& p \prec p \vee q \\
& p: \prec: p \cdot p \vee q
\end{aligned}
$$

$[(1),(2)]$

$p \cdot p \vee q: \prec: p$

17.01-17.4 is easily proved except $17.01^{\prime}$ and $17.12^{\prime}$,

$\dagger 17.01^{\prime}$

$\dagger 17.12^{\prime}$

$$
\sim(p \prec \sim q) \cdot \prec \cdot p \circ q
$$$$
\sim(p \circ \sim q) \cdot \prec \cdot p \prec q
$$

*17.5 is considered later. 17.51 and 17.52 are proved against the statement of Vredenduin as follows :

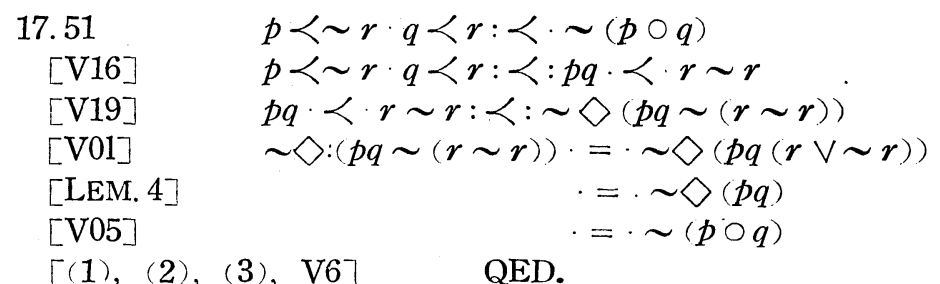

\section{$17.52 \quad p \prec q \cdot p \prec \sim q: \prec \cdot \sim(p \circ p) \quad[17.5, q / p p / q]$}

$17.53-17.71$ are all proved except 17.592, 17.7 and 17.71, where $17.7,17.71$ need not to be considered, because of 19.692 .

* 17.592

$$
p \circ p \cdot \prec: p \circ q \cdot \vee \cdot p \circ \sim q
$$

18. $1-18.92$ are proved except the following propositions :

$+18.1^{\prime}$

† 18. $12^{\prime}$

$\sim(p \prec \sim p) \cdot \prec \cdot \diamond p$

$+18.13^{\prime}$

$\sim \diamond p \cdot \prec \cdot p \prec \sim p$

$+18.14^{\prime}$

$\sim(\sim p<p) \cdot \prec \cdot \diamond \sim p$

$+18.2^{\prime}$

$+18.3^{\prime}$

$\sim \diamond \sim p \cdot<\cdot \sim p \prec p$

$+18.31^{\prime}$

$\sim(p \sim q \cdot 0 \cdot p \sim q) \cdot \prec \cdot p \prec q$

$+18.35^{\prime}$

$\sim(p \prec \sim q) \cdot \prec p \circ q$

$+18.36^{\prime}$

$\sim(p \circ q) \cdot\langle\cdot p<\sim q$

$+18.61$

$\sim(p q \cdot \prec \sim r)<\diamond(p q r)$ etc.

$\dagger 18.7^{\prime}$

$\sim(q r s \cdots \prec \cdot \sim p) \cdot \prec \cdot \diamond(p q r s \cdots)$ etc.

19. 02

$\sim \diamond \sim p \cdot p q<r: \prec \cdot q<r$

19.51

$\sim \diamond \sim(p \supset q) \cdot \prec \cdot p \prec q$

[16.33]

19. 451 are easily deduced.

[V15]

$$
\begin{aligned}
& p \prec r \cdot \prec \cdot p q \prec r \\
& p \prec r=p \prec p r \\
& p \prec p r \cdot \prec \cdot p q \prec p r q
\end{aligned}
$$


[16.33]

$$
p q \prec r=\cdot p q \prec p q r
$$

$[(1),(2),(3)]$

QED.

$19.52 q \prec r \cdot \prec \cdot p q \prec r \quad[19.51,12.15]$

$19.5 p \prec r \cdot \vee \cdot q \prec r: \prec: p q \prec r \quad$ [LEM. 2,19.51, 19.52,13.31]

$19.48 p \prec q: \prec: p \cdot \prec q \vee r \quad[19.51,12.44]$

19.49 $p \prec r: \prec: p \cdot \prec \cdot q \vee r \quad[19.52,12.44]$

19.46 $p \prec q \cdot \vee \cdot p \prec r: \prec: p \cdot \prec \cdot q \vee r \quad[19.48,19.49$ LEM. 2$]$

$19.47 p \prec q \cdot \vee \cdot p \prec r \cdot \vee \cdot \sim q \prec r: \prec: p \cdot \prec \cdot q \vee r$

[19.48] $\quad \sim q \prec r: \prec: \sim q \cdot \prec \cdot r \vee \sim p$

[LEM. 5]

$$
\sim q \prec \prec \cdot r \vee \sim p:=: p \cdot \prec \cdot r \vee q
$$

$[(1),(2), 13.11] \sim q \prec r: \prec: p \cdot \prec \cdot q \vee r$

$[(3), 19.46$, LEM. $2,13.31]$ QED.

19.57

$$
\begin{aligned}
& p \cdot q \sim q:=: q \sim q \\
& q \sim q \cdot \prec: p \cdot q \sim q \\
& p \cdot q \sim q: \prec \cdot q \sim q
\end{aligned}
$$

[LEM. 3]

[12.17]

$[(1),(2)]$

QED.

19.58 (= second part of LEM. 4)

19.6 is identical with V15, and 19.61 is a special case of V16.

19. $62 p \prec q r: \prec: p \prec q \cdot p \prec r$

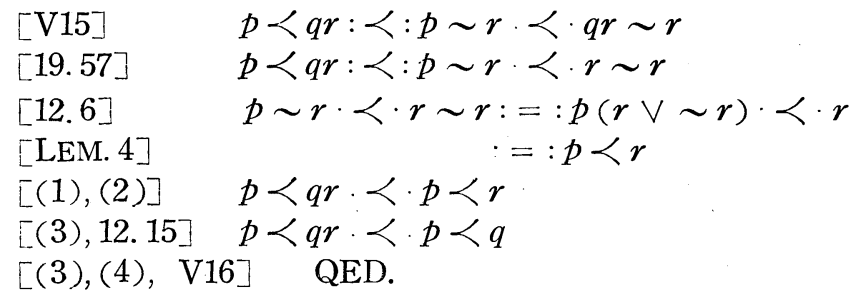

19.63 $p \prec q r:=: p \prec q \cdot p \prec r . \quad[19.61,19.62\rfloor$

19.64 is identical with LEM. 1.

19. 65 is a special case of LEM. 2 .

$19.66-19.682$ are easily deduced from the above formulas.

* 19.69 $p \cdot \circ \cdot q \vee r: \prec: p \circ q \cdot \vee \cdot p \circ r$ is considered later.

$19.692-19.92$ are easily proved except the following parts :

* 19.692' $(=19.69), * 19.7^{\prime}(=17.592)$

$* 19.71^{\prime} \diamond p \cdot \prec: \diamond(p q) \cdot \vee \diamond \diamond(p \sim q)$

$\dagger 19.72^{\prime}-1 \sim \diamond p: \prec: p \prec \sim q \cdot p \prec q$

$\dagger 19.72^{\prime}-2 \sim(p \circ q) \sim(p \circ \sim q): p \prec \sim q \cdot p \prec q$

* $19.72^{\prime}-3 \sim(p \circ q) . \sim(p \circ \sim q):\langle: \sim \diamond p$

$\dagger 19.73^{\prime} \sim \diamond \sim p \prec: q<p \cdot \sim q \prec p$

$\dagger 19.74, \dagger 19.75$

$\dagger 19.76 \sim(p<q) \cdot \prec \cdot \diamond p$

$19.77 \sim(q<p) \cdot \prec \cdot \diamond \sim p$

* 19. $8^{\prime} \quad \sim>p \sim \diamond q \cdot \prec \cdot \sim \diamond(p \vee q)$

* 19. 81' $\sim \diamond \sim p \sim \diamond \sim q \cdot<\sim \diamond \sim(p q)$ 
$\begin{array}{ll}* 19.82^{\prime} & \diamond(p \vee q) \cdot \prec: \diamond p \cdot \vee \cdot \diamond q \\ \dagger 19.83 & \sim \diamond p \sim \diamond q \cdot \prec \cdot p=q \\ \dagger 19.84 & \sim \diamond \sim p \sim \diamond \sim q \cdot \prec \cdot p=q\end{array}$

2. *-Propositions. (i) It is obvious that 17.592, 19.71' and $19.72^{\prime}-3$ are deducible from one another, and $19.8^{\prime}, 19.81^{\prime}$ and $19.82^{\prime}$ are deducible from one another.

(ii) $19.82^{\prime}$ is deducible from $19.71^{\prime}$ :

$\left[19.71^{\prime}\right] \diamond(p \vee q) \cdot\langle: \diamond(p \vee q \cdot q) \cdot \vee \cdot \diamond(p \because q \cdot \sim q)$

$[16.35,16.72] \diamond(p \vee q \cdot q) \cdot \vee \cdot \diamond(p \vee q \cdot \sim q)$

$:=: \diamond q \vee \diamond \cdot p \sim q \cdot \vee \cdot q \sim q$

[LEM. 1] $\diamond(p \sim q) \cdot \vee \cdot \diamond q: \prec: \diamond p \cdot \vee \cdot \diamond q$

$[(1),(2),(3), \mathrm{V} 6] \diamond(p \vee q) \cdot \prec: \diamond p \cdot \vee \cdot \diamond q$ QED.

(iii) 19.69 is deducible from $19.82^{\prime}$ :

$\left[19.82^{\prime}\right] \diamond(p q \cdot \vee \cdot p r):\langle: \diamond(p q) \cdot \vee \cdot \diamond(p r)$

[16.72] $p q \cdot \vee \cdot p r:=: \mathrm{p}(q \vee r)$

$[(1),(2), \mathrm{V} 05] \quad p \cdot \circ \cdot q \vee r: \prec: p \circ q \cdot \prec \cdot p \circ r$ QED.

(iv) 17.592 is deducible from 19.69. [LEM. 4, 12.7]

(v) 17.5 is deducible from 19.81':

[16.35] $p r:=:(p r \vee q)$ thr

[16.72] : : : pr $\vee p q r$

[12.1] $\sim \diamond(p r) \cdot \prec \cdot \diamond(p r \cdot \vee \cdot p q r)$ $\sim \diamond(q \sim r) \cdot \prec \cdot \diamond(q \sim r \cdot \vee \cdot p q \sim r)$

$[(1),(2), \mathrm{V} 16] \sim \diamond(p r) \sim \diamond(q \sim r) \cdot \prec \cdot \diamond(p r \cdot \vee \cdot p q r)$

[19.81] $\sim \diamond(p r \cdot \vee q p r) \sim \diamond(q \sim r \cdot \vee \cdot p q \sim r)$

$$
:=: \sim \diamond(p r \cdot \vee \cdot p q r \cdot \vee \cdot q \sim r \cdot \vee \cdot p q \sim r)
$$

$[16.72]:=: \sim \diamond(p r \cdot \vee \cdot q \sim r \cdot \vee \cdot p q(r \vee \sim r))$

[LEM. 4] : : : $\sim \diamond(p r \cdot \vee \cdot q \sim r \vee \cdot p q)$

$[19.82] \quad:=: \sim \diamond(p r \cdot \vee q \sim r) \sim \diamond(p q):=: \sim \diamond(p r)$

$$
\sim \diamond(q \sim r) \sim \diamond(p q)
$$

$[(3),(4)] \sim \diamond(p r) \sim \diamond(q \sim r) \prec \sim \diamond(p r) \sim \diamond(q \sim r) \sim \diamond(p q)$

[16.33] $\sim \diamond(p r) \sim \diamond(q \sim r) \cdot \prec \sim \diamond(p q)$

[V05] $\sim(p \circ r) \sim(q \circ \sim r) \cdot \prec \cdot \sim(p \circ q)$ QED.

(vi) $19.7^{\prime}$ is deducible from $17.5[17.5, p / q q / r, 12.44]$

Hence, every $*$-proposition is deducible from one another in Vredenduin's system, but we can show that they are not deducible from his system. In order to deduce them we translate V18 into a new stronger postulate V18;

V18' $\diamond(p \sim q) \cdot \diamond p: \prec \diamond \diamond q$

If we designate this system by $\mathrm{V}_{2,}$, then in $\mathrm{V}_{2}$, we can deduce V18 and all the $*$-propositions which have no paradoxical structure, and also Vredenduin's aim is attained as will be shown later. 


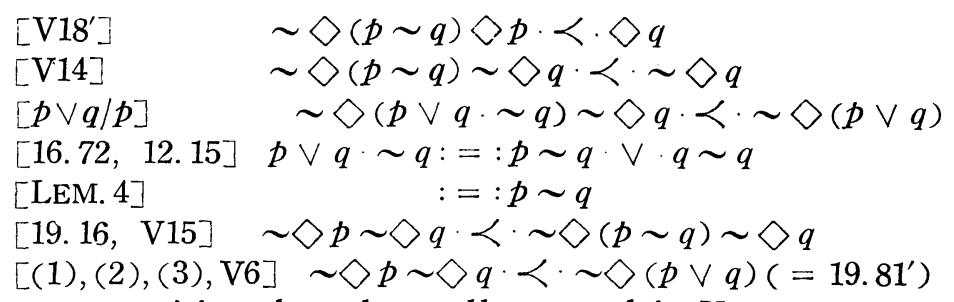

Hence, *-propositions have been all asserted in $\mathrm{V}_{2}$.

3. F-propositions and certain properties of the system $V_{2}$. We next consider about †-propositions, which seem to have more or less evidently paradoxical structures. As Vredenduin shows the independency of 19.74 and 19.75, of his system, we can show it in his way that none of $\dagger$-propositions can be deduced in this system $V_{2 .}$. As he states, all the assumptions of the system $V_{2}$ are altered to asserted propositions of the system S2 if the $\diamond$-symbols are omitted. Effectively, postulate V18' are then altered to $\sim(p \sim q) \cdot p: \prec q$. In the system S2, $\sim(p \sim q) p:=:$ $\sim p \vee q \cdot p:=: \sim p p \cdot \vee \cdot q p:=: p q$, and $p q \prec q$ are asserted, hence that is asserted. By Vredenduin's method we can show that 18.1', 19.61, 19.75, 19.84 are all independent of the system $\mathrm{V}_{2 .}{ }^{6}$ ) On the other hand, we can deduce any of $18.1^{\prime}, 19.61,19.75,19.84$ from $\dagger 11.01^{\prime} \sim \diamond(p \sim q)$. $\prec p \prec q$ or from any of the remainders of $\dagger$-propositions in the system $V_{2}$, then every paradoxical proposition is independent.

Further, the following fact is to be noticed: Halldén Sören shows ${ }^{7}$ ) that certain analogues of the paradoxes are deducible in SI, in which the consistency postulate regarded as the cause of the paradoxes is independent, namely

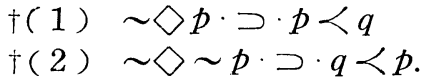

It can easily be shown in such a way as abjve, that (1) and (2) are not deducible in the system,$V_{2}$. ((1); $p=3, q=2,(2), p=2, q=1$, in

6) $18.1^{\prime} \cdots \cdots \sim(p \prec \sim p) \cdot \prec \cdot p$

$18.61 \cdots \cdots \sim(\sim p) \cdot p q<r:<\cdot q<r \quad$ (2)

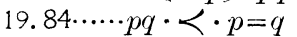

(1)

Lewis and Langford give the following normal S2-matrix '(S. L., p. 443, Group I)

\begin{tabular}{|c|c|c|c|c|c|}
\hline$p q$ & 1234 & $\sim p$ & 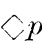 & $p<q$ & 1234 \\
\hline 1 & 1234 & 4 & 1 & 1 & 2444 \\
\hline 2 & 2244 & 3 & 1 & 2 & 2244 \\
\hline 3 & 3434 & 2 & 1 & 3 & 2424 \\
\hline 4 & 4444 & 1 & 3 & 4 & 2222 \\
\hline
\end{tabular}

Every proposition asserted in S2 has one of the designated values 1 and 2 . Now choose $p=2$, then (1) has the value 4, thoose $p=2, q=1$ and $r=2$, then (2) has 4; choose $p=1$ and $q=2$, then (3) has 4. Hence (1), (2) and (3) are independent of $\mathrm{S} 2$.

7) Halldés Söres, A note concerning the paradoxes of strict implication, Journ. of Symbolic Logic, vol. 13 (1948), pp. 138-139. 
Group 1. )

THEOREM 1. Every asserted proposition of S2 in Symboiic Logic is asserted in the system $V_{2}$ except $\dagger$-propositions which are not deducible in $\mathrm{V}_{\mathbf{2}}$.

The following theorem are evident by the comparison with the assumptions of the systems $\mathrm{S} 2$ and $\mathrm{V}_{2}$.

THEOREM 2. Every asserted proposition of $\mathrm{V}_{2}$ is also asserted in S2.

THEOREM 3. The system $\mathrm{V}_{2}$ has infinitely many complete extensions ${ }^{8}$.

This is easily shown by the Theorem 2 and by the fact that the system S2 has infinitely many complete extensions. ${ }^{8)}$

THEOREM 4. The number of irreducible molalities ${ }^{9}$ in the system $\mathrm{V}_{2}$ is infinite.

The proof is trivial by the Theorem 2 and by the fact that there are an infinite number of irreducible molalities in the system $\mathrm{S}^{10}$ )

II. Relations among Lewis's system, Emch's and Vredenduin's.

Designate the following system by $\mathrm{S} 2^{\circ}$, which are obtained when the primitive symbol $\diamond$ is altered to symbol $\bigcirc$. Let us add to the system $V_{2}$ a definition

$$
\text { V06 } O p=\sim \sim(p \prec \sim p) \text {. }
$$

Designate Emch's system by $\mathrm{E}_{2}$, which are obtained when we translate his symbol $\infty$ (logical implcation) into $\prec, \prec$ (strict implication) into $<$, ugical equivalence inio $=$, and strict equivalence into $I$ in Emch's system ${ }^{15}$,

Emch's system $E_{2}$ :

Undefined ideas; Elementary propositions $p, q, r$, etc., negation $\sim p$, product $p q$ or $\dot{p} q$, possibility $\diamond p$, consistency $\bigcirc p$, and equivalence $p=q$.

Postulates ;

L1 $\quad p q \prec q p$

L2 $\quad p \prec p p$

L3 $\quad(p q) r \prec p(q r)$

L4 $\quad p \prec \sim(\sim p)$

L5 $\quad p \prec q \cdot q \prec r: \prec \cdot p \prec r$

L6 $\quad p \cdot p \prec q: \prec q$

L7 $\diamond p \cdot \diamond q \cdot \prec \cdot \bigcirc \bigcirc q$

L8 $\diamond(p q) \cdot \prec \cdot \diamond p$

Definitions :

$$
\begin{array}{ll}
\text { L01 } & p \vee q=\cdot \sim(\sim p \sim q) \\
\text { L02 } & p \prec q=\sim \sim(p \sim q) \\
\text { L03 } & p=q=p<q \cdot q<p \\
\text { (L04) } & p<q=\sim \sim \diamond(p \sim q) \\
\text { (L05) } & p I q=\cdot p<q \cdot q<p \\
\text { L06 } & p \supset q=\sim(p \sim q) \\
\text { L07 } & p \equiv q=\cdot p \supset q \cdot q \supset p
\end{array}
$$

8) Mckinser, op. cit., the ffirst paper.

9) W. T. Parry, Modalities in the survey system of strict implication, Journ. of Symbolic Logic, vol. 4 (1939), p. 144.

10) Mckinsey, op. cit., the second paper.

11) Emch had an attempt such that the logical implication coincides with the usual deducibility, then there arises no essential absurdity though we alter the sf $\mathrm{mbol}$. 
L11 $\quad p \prec \diamond p$

L12 $\bigcirc(p q) \cdot \prec \cdot \bigcirc$

L13 $\diamond(p q) ; \prec: \diamond(p \sim r) \cdot \vee \cdot \diamond(r q)$

L14 Substitution (a)

L15 Substituion (b)

L16 Adjunction

L17 Inference

$q p \prec p$ is deducible in $\mathrm{E}_{2}$, and the other assumptions of $\mathrm{S} 2^{\circ}$ are included in one of $E_{2}$, then the following theorem is shown:

THEOREM 5. The asserted propositions in $\mathrm{S} 2^{\circ}$ are all asserted in the system $\mathrm{E}_{2}{ }^{12)}$.

Next, we show that the systems $\mathrm{E}_{2}$ and $\mathrm{V}_{2}$ are equivalent. Designate the corresponding proposition of $\mathrm{S} 2^{\circ}$ to one of $\mathrm{S} 2$ by the same number having the sign ${ }^{\circ}$ at the shoulder.

1. $\mathrm{V} 1, \mathrm{~V} 3-\mathrm{V} 12, \mathrm{~V} 17, \mathrm{~V} 01-\mathrm{V} 04$ are included in the assumptions of the system $\mathrm{E}_{2}$ and V2 is easily proved in $\mathrm{E}_{2}$. V13, V14, V15, and V16 are proved in S2 and invariant by the above translation, then they are proved also in the system $\mathrm{E}_{2}$ by Theorem 5 .

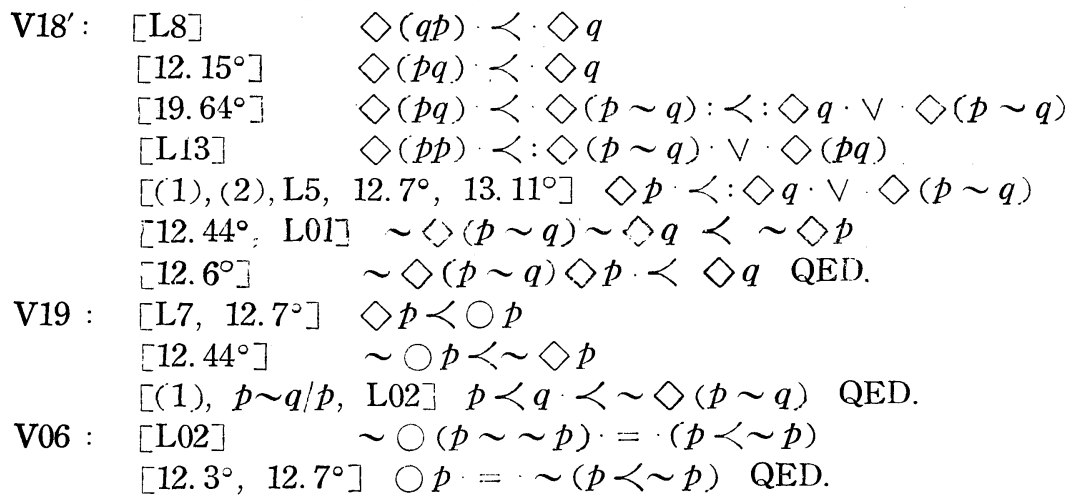

2. L01. L03, L06, L07, L1 - L6, L8, L11, L14- L17 are included in the assumptions of the system $\mathrm{V}_{2}$.

L02: $[\mathrm{V} 06,12.3,12.44] \sim \bigcirc p=p \prec \sim p$

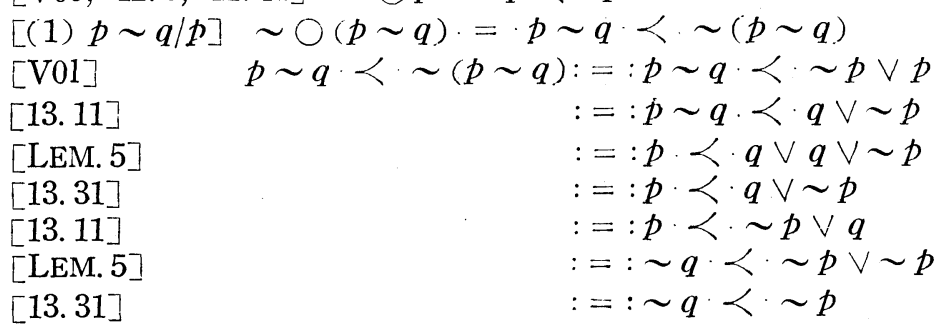

12) This was shown by Lewis, op. cit., 3) 
[12.44]

$$
\begin{gathered}
:=: p \prec q \\
\sim(p \sim q)=\cdot p \prec q \text { QED. }
\end{gathered}
$$

$[(1),(2), \mathrm{V} 16] \diamond p \diamond q \prec \sim(p \prec \sim p) \sim(q \prec \sim q)$

[V06] $\quad \diamond p \diamond q \prec \bigcirc p \bigcirc q$ QED.

L12 : [V15] $p \prec \sim p \cdot \prec: p p q \cdot \prec \cdot \sim p q$

$[12.7,19.57] p p q \cdot \prec \cdot p p q:=: p q \cdot \prec p \sim p$

[12.6] : $:: p(p \vee \sim p) \cdot \prec \cdot \sim q$

[LEM. 4]

$[(1),(2)]$

$$
p \prec \sim p: \prec p \prec \sim q
$$

$$
:=: p \prec \sim q
$$

$[\mathrm{V} 06,12.44, \mathrm{~L} 02] \bigcirc(p q) \prec \bigcirc p$ QED.

L13 is easily proved by 17.5 .

Hence the following theorem has been established;

THEOREM 6. The asserted propositions of the system $\mathrm{V}_{2}$ are all asserted in the system $\mathrm{E}_{2}$, and vice versa.

Thus we have concluded by the theorems 1,2, 5 and 6 that ( $i$ ) the system $S 2$ includes the systems $V_{2}$ and $E_{2}{ }^{13)}$, (ii) the systems $V_{2}$ and $E_{2}$ are equivalent, and (iii) the systems $V_{2}$ and $E_{2}$ include the system $S 2^{\circ}$, hence, both in $V_{2}$ and in $E_{2}$, we can deduce not only the propositions which have been asserted in $\mathrm{S} 2$, but also deducible one in $\mathrm{S} 2$, if they are invariant by the translation $\diamond$ into $\bigcirc^{14)}$.

III. Certain extensions of system $V_{2 .}$ Becker $^{15}$ and the others ${ }^{16)}$ made attempts to construct extensions of the system S2 or S3 in view of modality. Whether can we hold the analogous extensions concerning to the system $V_{2}$ without loss of Vredenduin's purpose or not, and how many modalities have they? As Tang ${ }^{17)}$ and Parry ${ }^{18)}$ show in S2, we can deduce the following lemmas in the system $\mathrm{V}_{2}$.

LEMMA 6. $p \prec q \cdot=\cdot p=p q$

[19.62, V2, V6] $p \cdot \prec p q \cdot \prec \cdot p \prec p$

[19.51]

$p<p \cdot \prec \cdot p q<p$

[(1), (2), V6] $\quad p \prec p q \cdot \prec \cdot p q \prec q$

13) We must, of course, add the Definition $\bigcirc p .=\sim \sim(p<\sim p)$ to the system S2.

14) If, in $V_{2}$, we prove only the propositions used in the proof of $V_{2} \rightarrow E_{2}$, certain proofs in I, of course, are unnecessary.

15) O. Becken, Zur Logik der Modalitäten, Jahrbuch für Philosophie und phänomenologische Furschung, vol. 11(1930), pp 497-548.

15) Parry, op. cit. and C. W. Churchman, On finite and infinite modal systems, Journ. of Symbolic Logic, vol.3 (1938), pp. 77-82

17) T. C. TANG, The theorem $p<q .=\cdot p q=p$ and Huntington's relation between Lewis's strict implication and Boolean algebra. Bull. Amer. Math. Soc., vol. 42 (1936), pp. 743-746.

E. V. Huntington, Postulates for assertion, conjunction, negation, and equality., Proceed. of Arts and Sciences, vol. 72 (1938), pp.1-44, Theorem 97 (p. 24)

18) Parry, op.cit. 
[16.33]

[V2]

$[(3),(4), \mathrm{V} 02]$

$$
\begin{aligned}
& p \prec p q \cdot \prec: p \prec p q \cdot p q \prec p \\
& p \prec p q \cdot p q \prec p: \prec \cdot p \prec p q \\
& p=p q \cdot=p \prec \prec p q \\
& p=p q=p \prec q \text { QED. }
\end{aligned}
$$

LEMMA 7. If $p \prec q$ has been asserted, then $\diamond p \prec \diamond q, \sim \diamond q \prec \sim \diamond p$, and $\sim \diamond \sim \diamond p \prec \sim \diamond \sim \diamond q$ may be asserted.

[Hyp., LEM. 6] $p=p q$

[V8] $\quad \diamond(q p) \cdot \prec \cdot \diamond q$

$[(1),(2)]$

$\diamond p \cdot \prec \cdot \diamond q$

[12.44] $\quad \sim \diamond q \cdot \prec \cdot \sim \diamond p$

[(3)] $\quad \sim \diamond \sim \diamond p \cdot \prec \cdot \sim \diamond \sim \diamond q$ QED.

LEMMA 8. $\sim \diamond \sim p \cdot \prec \sim \diamond \sim \diamond p$. [18.41, LEM. 7]

1. Designate by $\mathrm{V}_{4}$ the system deduced from the set $\left(\mathrm{V}_{22}\right.$ and the following postulate $\mathrm{C} 10$ ).

$\mathrm{C} 10$

$$
\sim \diamond \sim p \cdot \prec \cdot \diamond \sim \sim \diamond \sim p
$$

Then the following lemmas can be deduced in $\mathrm{V}_{4}$.

LEMMA 9. $\diamond_{n} p=\diamond p$, where by " $\oslash_{n} p$ " we mean the formula which is formed by putting $n$ " $\diamond$ " symbols in front of $p$.

$[\mathrm{C} 10,12.3] \sim \diamond \sim p \cdot \prec \cdot \sim \diamond_{2} \sim \mathrm{p}$

[12.44] $\diamond_{2} \sim p \cdot \prec \cdot \diamond \sim p$

[12.3] $\quad \diamond_{2} p \cdot \prec \cdot \diamond p \quad$ QED.

LEMMA 10. $\sim \diamond p \cdot \prec \cdot \diamond \sim \diamond \sim \diamond p$

[LEM. 8, $\sim \diamond p / p] \sim \diamond \sim \sim \diamond p<\sim \diamond \sim \diamond \sim \diamond p$

[LEM. 9] $\quad \sim \diamond p \prec \sim \diamond \sim \diamond \sim \diamond p$ QED.

LEMMA 11. $\sim \diamond \sim \diamond p=\sim \sim>\sim>>\sim \diamond p$

[LEM. 10, LEM. 7] $\sim \diamond \sim \diamond \sim \diamond \sim \diamond p \cdot \prec \cdot \sim \diamond \sim \diamond p$

[LEM. 10, $\diamond p / p] \sim \diamond \sim \diamond p \cdot \prec \sim \diamond \sim \diamond \sim \diamond \sim \diamond p$

$[(1),(2)]$

QED.

LEMMA $12 . \sim \diamond \sim \diamond \sim \diamond \sim p \cdot \prec \cdot \diamond \sim \diamond \sim p$ [18.42], $\sim \diamond \sim p \cdot \prec \sim \diamond \sim$ $\diamond \sim \diamond \sim p$ [LEM. 10] $\sim \diamond \sim \diamond \sim \diamond \sim p<\sim \diamond \sim \diamond p$ [LEM. 7, 18.42], $\diamond \sim \diamond \sim$ $p \prec \diamond \sim \diamond \sim \diamond \dot{p} \quad[$ LEM. 8,7$], \sim \diamond \sim \diamond p<\diamond \sim \diamond \sim \diamond p[\mathrm{~V}$ 17], $\diamond \sim \diamond \sim \diamond$ $p<\diamond p$ [LEM. 10] and the propositions deduced from these propositions by 12.44 .

This system $V_{4}$ is evidently included in $\mathrm{S} 4$ by Theorem 2 , and it was proved by Parry ${ }^{19}$ ) that these fourteen modalities cannot be further reduced in S4.

THEOREM 7. The propositions asserted in $\mathrm{V}_{4}$ are all asserted in S4 and the number of irreducible modalities in $V_{4}$ is fourteen.

2. Designate by $V_{4 \cdot 5}$ the system deduced from the set $\left(V_{4}\right.$ and a new postulate $\mathrm{C} 4.5$ ).

C $4.5 \quad \sim \diamond \sim \diamond \sim \diamond p \prec \sim \diamond p$

LEMMA 13. $\sim \diamond \sim \diamond \sim \diamond p=\cdot \sim \diamond p, \diamond \sim \diamond \sim \diamond p=\cdot \diamond p$,

19) Parry, op.cit. 


$$
\diamond \sim \diamond \sim \diamond \sim p=\cdot \diamond \sim p, \sim \diamond \sim \diamond \sim \diamond \sim p=\cdot \sim \diamond \sim p .
$$

Hence the fourteen modalities are reduced to ten in $\mathrm{V}_{4 \cdot 5}$.

THEOREM 8. The number of irreducible modalities in $\mathrm{V}_{4 \cdot 5}$ is at most ten.

3. Designate by $\mathrm{V}_{4 \cdot 51}$ the system deduced from the set $\left(\mathrm{V}_{4}\right.$ and a new postulate $\mathrm{C} 16$ ).

$\mathrm{C} 16$

$$
\diamond \sim \diamond \sim p=\sim \diamond \sim \diamond p
$$

Then,

$$
\sim \diamond \sim p \prec \sim \diamond \sim \sim_{p} \diamond p \prec \diamond p, \quad \sim \diamond p \prec \diamond \sim \diamond p \prec \sim \diamond p .
$$

In the corresponding system of Parry, including this system $V_{4 \cdot 51}$, the above eight modalities can not be further reduced.

THEOREM 9. The number of irreducible modalities in $V_{4^{*} 51}$ is eight.

4. Designate by $\mathrm{V}_{5}$ the system deduced from the set $\left(\mathrm{V}_{2}\right.$ and a new postulate $\mathrm{C} 11$ ).

C 11

$$
\diamond p \cdot \prec \cdot \sim \sim \diamond p
$$

As in S5, it is easily proved that, C11 is deducible from $\mathrm{C} 10$ and $\mathrm{C} 12$ $p \cdot \prec \sim \diamond \sim \diamond p$ in $\mathrm{V}_{5}$, and vice versa. $\mathrm{C} 4.5$ is dedubible from $\mathrm{C} 11$ in $\mathrm{V}_{5}$, hence $\mathrm{V}_{4}$ and $\mathrm{V}_{4 \cdot 5}$ are included in $\mathrm{V}_{5}$.

LEMMA 14. $\diamond p=\sim \diamond \sim \diamond p$

$[18.41, \sim \diamond p / p] \sim \diamond \sim \diamond p \cdot \prec \sim \diamond p$

$[12.3] \quad \sim \diamond \sim \diamond p \cdot \prec \cdot \diamond p$

$[(1), \mathrm{C} 11]$ QED.

Then. by Lemma 14 and the considerations in $\mathrm{V}_{4 \cdot 5}$. everv modalitv in $\mathrm{V}_{5}$ is reduced to one ut the tollowing six $\sim \vee \sim p \prec p \cdot \prec p ; \sim \diamond p \prec$ $\sim p \cdot \prec \cdot \diamond \sim p$.

On the other hand, this system $V_{5}$ is included in $\mathrm{S} 5$, and these six modalities can not be further reduced in $\mathrm{S} 5$, hence,

THEOREM 10. The propositions asserted in $\mathrm{V}_{5}$ are all asserted in S5, and the number of irreducible modalities in $V_{5}$ is six.

It is noticed that all these extensions of the system $V_{2}$, have no paradoxical $\dagger$-propositions as Vredenduin's system. If we omit the symbol $\diamond$ in $\mathrm{C} 11$ and $\mathrm{C} 16$, we have $p \cdot \prec \sim \sim p$ and $\sim p=\sim \sim p$ respectively, and they are deducible in S2.

THEOREM 11. Any paradox of $\dagger$-propositions can not be deduced in each extension $V_{4}, V_{4 \cdot 5}, V_{4 \cdot 51}, V_{5}$ of the system $V_{3}$.

The author expresses here his hearty thanks to Professor M. Ito to whom he has been indebted for his many valuable remarks and suggestions.

Mathematical Institute, TôHokU UnIveksitry 